\title{
Complex band structure under plane-wave nonlocal pseudopotential Hamiltonian of metallic wires and electrodes
}

\author{
M. G. Vergniory ${ }^{1}$, C. Yang ${ }^{1}$, A. Garcia-Lekue ${ }^{2}$ and Lin-Wang Wang ${ }^{1}$ \\ ${ }^{1}$ Computational Research Division, Lawrence Berkeley National Laboratory, California 94720, USA \\ ${ }^{2}$ Donostia International Physics Center (DIPC) , Basque Country, Spain
}

(Dated: July 17, 2009)

\begin{abstract}
We present a practical approach to calculate the complex band structure of an electrode for quantum transport calculations. This method is designed for plane wave based Hamiltonian with nonlocal pseudopotentials and the auxiliary periodic boundary condition transport calculation approach. Currently there is no direct method to calculate all the evanescent states for a given energy for systems with nonlocal pseudopotentials. On the other hand, in the auxiliary periodic boundary condition transport calculation, there is no need for all the evanescent states at a given energy. The current method fills this niche. The method has been used to study copper and gold nanowires and bulk electrodes.
\end{abstract}

PACS numbers: 71.15.-m, 71.15.Dx, 73.20.At, 73.22.-f

\section{INTRODUCTION}

As the traditional CMOS device is reaching its length limit, there is a flurry of research activities to find alternative materials, designs, or even completely new systems (like the molecular electronics, and nanotubes) to replace the traditional Si based CMOS. Due to the small scales of all these proposed new devices, quantum mechanical transport calculations are essential in their simulations. The elastic quantum mechanical transport can be calculated using nonequilibrium Green's function technique, or via the scattering states calculations. In both approaches, the complex k-point band structure of the electrodes, which includes both the running wave states and evanescent states, are needed. In practice, the computation of the electrode complex band structure can be a big part of the overall transport calculation. It might not be so straightforward for many of the electrodes, especially when the electrode is not just a supercell of the bulk system. The computation becomes especially challenging when plane wave basis set is used, and nonlocal pseudopotential exist in the Hamiltonian, as there is no direct methods to yield all the evanescent states for a given energy level. On the other hand, in our new auxiliary periodic boundary condition approach for quantum transport calculation it is not necessary to get all the evanescent states for a given energy in order to get the scattering states. Thus, it is desirable to find new practical ways to calculate the complex band structures.

There are long established methods for complex band structure calculations. Suppose $H(\mathbf{k})$ is the Hamiltonian matrix under a basis set with Bloch vector $\mathbf{k}$, here $\mathbf{k}$ has both real and imaginary parts. The complex band structure problem can be posed as one that seeks a value of $\mathbf{k}$ for which Hamiltonian $H(\mathbf{k})$ has a prescribed eigenenergy $E$. A straightforward approach to tackle this problem is to solve $\operatorname{det}|H(\mathbf{k})-E|=0$ for $\mathbf{k}$. For example, one can scan a range of values of $\mathbf{k}$ until $\operatorname{det}|H(\mathbf{k})-E|$ becomes negligibly small. However, this approach might not be very efficient because the evaluation of $H(\mathbf{k})$ for each new $\mathbf{k}$ is time consuming, and $\mathbf{k}$ is complex (computationally is a 2 dimensional variable). Using a simple tight-binding model, we may obtain an analytical expression for $H(\mathbf{k})$. In this case, it can be shown that $\operatorname{det}|H(\mathbf{k})-E|$ is an Nth degree polynomial of $\xi=\exp \left(i k_{z} a\right)$. Here complex $k_{z}$ is the $\mathrm{z}$ (the direction of the electrode for which the band structure is calculated) component of $\mathbf{k}$, and $a$ is an interlayer atomic distance in the $\mathrm{z}$ direction. The degree $\mathrm{N}$ is proportional to the number of neighboring layers in the tight binding model multiplied by the atomic orbitals in those layers. In practice, $\mathrm{N}+1$ determinants are first calculated. This calculation yields $\mathrm{N}+1$ polynomial coefficients from which the roots of the complex polynomial are then searched. ${ }^{1}$ However, this method becomes numerically unstable when the dimension of the system is large.

In another method, ${ }^{2}$ the original complex band structure eigenvalue matrix equation $H(\mathbf{k}) C=E C$ is expanded into a bigger eigenvalue matrix equation many times of its original size. For example, in a tight-binding formalism, due to the analytical expression of the role of $k_{z}$ inside $H(\mathbf{k})$, the original equation can be rewritten as $H_{2 m}(E) C_{2 m}^{\prime}=\exp \left(i k_{z} a\right) C_{2 m}^{\prime}$. Here $m$ is the number of neighboring layers in the tight-binding Hamiltonian, and the subscript $2 m$ indicates that the dimension of the matrix $H_{2 m}(E)$ is $2 m$ times the original matrix $H(\mathbf{k})$. To obtain $H_{2 m}(E)$, the inverse of matrices $H^{(\sigma)}-E$ $(\sigma=-m, m)$ must be first calculated. Because these matrices may become nearly singular, the inversion of these matrices must be done carefully. The generalized matrix equation $H_{2 m}(E) C_{2 m}^{\prime}=\exp \left(i k_{z} a\right) C_{2 m}^{\prime}$ is then diagonalized, and the corresponding complex eigenvalues will give us the corresponding $k_{z}$. The same thing can be worked out for plane wave pseudopotential Hamiltonian without the nonlocal part. In a plane wave Hamiltonian without nonlocal potential, the $\mathbf{k}$ only appear in the kinetic energy operator, thus there are terms like $k_{z}$ and $k_{z}^{2}$. As a result, the matrix eigenvalue equation can be rewritten as $H^{\prime}(E) C=k_{z} C$, where the dimension of $H^{\prime}(E)$ is twice of that in the original plane wave basis set. Again, 
the complex eigenvalue of this equation gives directly the complex value of $k_{z}$.

Unfortunately, such nice analytical expression for the role of $k_{z}$ does not exist when there is a nonlocal potential. As a result, none of the above techniques can be applied, except the direct evaluation of $\operatorname{det}|H(\mathbf{k})-E|$, which can be expensive to calculate and difficult to search for the zeros. In a paper by Choi and Ihm, ${ }^{3}$ a transfer matrix method was proposed to deal with the nonlocal pseudopotential. In this method, the wave function for a given energy $E$ is propagated following the solutions of the Schrodinger's equation. The phase and amplitude of the wave function can be used to give the value of $k_{z}$. In a complicated band structure, the Bloch wave function boundary condition is enforced by a linear combination of many propagating wave functions. Such transfer matrix method relies on the local Laplacian operator in the $\mathrm{z}$ direction. Thus the nonlocal potential poses a challenge. In Ref. ${ }^{3}$, this difficulty is resolved by a linear combination of general solutions of the Schrodinger's equation (homogeneous linear equation) without the nonlocal potential term, plus the particular solutions of the nonhomogeneous linear equation with the nonlocal potential as the nonhomogeneous part. For every nonlocal potential components (atoms and angular momentum), one needs to solve the corresponding particular solution. Thus the total calculation can be expensive and messy for large systems. There are also typical issues for numerical stability for the transfer matrix techniques. Moreover, by expanding the wave function in $(x, y)$ cross section using plane wave basis while treating $z$ direction with real space transfer matrix method, we do not have a pure plane wave basis set method. Many of the above methods solve for all possible $k_{z}$ for a given $E$. That is necessary for many of the transport calculation methods. But in our new auxiliary periodic boundary condition transport calculation, only a few small $\operatorname{Im}\left(k_{z}\right)$ evanescent states are needed. This calls for development of new approaches to calculate the complex band structures.

In the last few years, we have developed a scattering state calculation method using purely plane wave basis set. $^{4-6}$ The scattering states are calculated via an auxiliary periodic boundary condition. The method transforms an open boundary condition scattering state problem into a conventional closed periodic boundary condition problem. As a result, the conventional numerical methods such as the conjugated gradient method can be used to solve the related wave functions (called system states). In order for this method to work, one has to decompose the system states in an electrode unit into the electron running wave and evanescent states for a given energy. Thus, we need the complex band structure of the electrode under the pure plane wave basis. However, we only need evanescent states with relativity $\operatorname{small} \operatorname{Im}\left(k_{z}\right)$. In most cases, the atoms of the electrodes are represented by nonlocal pseudopotentials.

In this paper, we present a simple procedure to calculate the complex band structure for a general plane wave non-local pseudopotential Hamiltonian. Instead of using sophisticated formalisms such as methods discussed above, the method we propose will be based on the original Hamiltonian $H(\mathbf{k})$. Instead of searching for the values of k's for a given $E$, we will search for $\operatorname{Im}\left(k_{z}\right)$ for a given $\operatorname{Re}\left(k_{z}\right)$, so the $\operatorname{Im}(E)$ is zero. The purpose of this paper is several fold: (1) to demonstrate that it is practical to use this simple approach to calculate the complex band structures of the typical electrodes used in transport calculations; (2) to present the computational scaling of diagonalizating a generalized matrix under the plane wave basis set using the PARPACK package $;^{7}$ (3) to show that how a simple anticrossing model can be used to aid the finding of $\mathbf{k}$ for yielding-real $\mathbf{E}$.

The paper is organized as follows. In Sec. II we describe the full $a b$ initio treatment of the band structure of a generic wire using a plane wave Hamiltonian. Within this section we will introduce the methodology to calculate the evanescent states. The computational method we use to calculate the complex band structure will be presented in Sec. III. In Sec. IV we will present our results of the complex band structure of 9 atoms unit-cell of $\mathrm{Cu}$ and a $3 \times 3$ electrode of $\mathrm{Au}$. We draw a few conclusions in Sec. IV. Unless otherwise stated, we use atomic units throughout the paper, i.e., $e^{2}=\hbar=m_{e}=1$.

\section{THE FORMALISM OF COMPLEX BAND STRUCTURE CALCULATIONS}

A conventional band structure is obtained by solving the Hamiltonian for a fixed real $\mathbf{k}$ vector, which yields the well known $E(\mathbf{k})$ relationship. For the complex band structure we consider a generalized Bloch state in real space,

$$
\phi_{\mathbf{k}}=U_{n, \mathbf{k}}(r) e^{i \mathbf{k r}-\kappa z}
$$

where $\mathbf{k}$ is the real part of the complex vector and $\kappa$ is the imaginary part of the wave vector. In this study, we will ignore the transverse real $\mathbf{k}$ component $\mathbf{k}_{\perp}$, thus the vector $\mathbf{k}$ will be in the $z$ direction, which is the direction of propagation of the electrons.

Our single particle plane wave Hamiltonian is written as,

$$
H=\left[-\frac{1}{2} \nabla^{2}+V(r)+V_{\text {nonloc }}\right]
$$

where $V_{\text {nonloc }}$ is the nonlocal part of the Hamiltonian. Then the Schrödinger equation becomes:

$$
H U_{n, \mathbf{k}}(r) e^{i \mathbf{k} \cdot \mathbf{r}-\kappa z}=E U_{n, \mathbf{k}}(r) e^{i \mathbf{k} \cdot \mathbf{r}-\kappa z} .
$$

Switching to the reciprocal space, the generalized Bloch states are written as,

$$
U_{n, \mathbf{k}} e^{i \mathbf{k} \cdot \mathbf{r}-\kappa z}=\sum_{\mathbf{G}} C_{n, \mathbf{k}} e^{i \mathbf{G r}} e^{i \mathbf{k} \cdot \mathbf{r}-\kappa z}
$$


In the reciprocal space we can define the following Hamiltonian matrix

$$
H_{\mathbf{G}, \mathbf{G}^{\prime}}^{\mathbf{k}}=\left\langle e^{i \mathbf{k r}+\kappa z} e^{i \mathbf{G r}}|H| e^{i \mathbf{k r}-\kappa z} e^{i \mathbf{G}^{\prime} \mathbf{r}}\right\rangle .
$$

Thus the generalized matrix Hamiltonian under the generalized plane wave basis $e^{i \mathbf{k r}+i \mathbf{G r}-\kappa z}$ becomes

$$
\begin{aligned}
H_{\mathbf{G}, \mathbf{G}^{\prime}}^{\mathbf{k}}=-\frac{1}{2}[i(\mathbf{G}-\mathbf{k})-\hat{z} \kappa]^{2} \delta_{\mathbf{G}, \mathbf{G}^{\prime}}+ \\
\quad V\left(\mathbf{G}-\mathbf{G}^{\prime}\right)+V_{\text {nonloc }}^{\mathbf{k}}\left(\mathbf{G}, \mathbf{G}^{\prime}\right) .
\end{aligned}
$$

The nonlocal part of the Hamiltonian can be calculated using the Kleinman and Bylander (KB) projectors. ${ }^{8}$ Mathematically, the angular dependent pseudopotential can be written as:

$$
\hat{V}_{\text {nonloc }}(r)=\sum_{l}|l\rangle \Delta V_{l}(r)\langle l|,
$$

where $|l\rangle$ is an angular momentum projection operator and $\Delta V_{l}(r)$ is the nonlocal potential for each angular momentum $l$.

Under the KB approximation, $\hat{V}(r)$ can be rewritten as

$$
\hat{V}_{\text {nonloc }}(r)=\sum_{l m} \frac{\left|\Delta V_{l} \phi_{l} Y_{l m}\right\rangle\left\langle\Delta V_{l} \phi_{l} Y_{l m}\right|}{\left\langle\phi_{l}\left|\Delta V_{l}\right| \phi_{l}\right\rangle}
$$

here $m$ is the azimuthal angular momentum, $Y_{l m}$ is the solid angle spherical harmonic and $\phi_{l}$ are the atomic radial pseudo wave functions for angular $l$. If we define the following projector,

$$
\Phi_{l}=\sum_{m} \frac{\left|\Delta V_{l} \phi_{l} Y_{l m}\right\rangle}{\sqrt{\left\langle\phi_{l}\left|\Delta V_{l}\right| \phi_{l}\right\rangle}}
$$

the nonlocal potential can then be written as

$$
\hat{V}_{\text {nonloc }}(r)=\sum_{l}\left|\Phi_{l}\right\rangle\left\langle\Phi_{l}\right| .
$$

Following Eq. (5) the KB nonlocal potential can be represented in $\mathbf{G}$ space as:

$$
\begin{aligned}
& V_{\text {nonloc }}^{\mathbf{k}}\left(\mathbf{G}, \mathbf{G}^{\prime}\right)= \\
& \sum_{l}\left\langle e^{i \mathbf{k r}+i \mathbf{G r}+\kappa z} \mid \Phi_{l}\right\rangle\left\langle\Phi_{l} \mid e^{i \mathbf{k r}+i \mathbf{G}^{\prime} \mathbf{r}-\kappa z}\right\rangle .
\end{aligned}
$$

Finally the eigenenergy can be solved from the following general eigenvalue equation:

$$
\sum_{\mathbf{G}^{\prime}} H_{\mathbf{G}, \mathbf{G}^{\prime}}^{\mathbf{k}} C_{n, \mathbf{k}}\left(\mathbf{G}^{\prime}\right)=E_{n, \mathbf{k}} C_{n, \mathbf{k}}(\mathbf{G})
$$

where $H_{\mathbf{G}, \mathbf{G}^{\prime}}^{\mathbf{k}}$ are elements of a complex non-Hermitian matrix. The size of this matrix for realistic systems can be as large as $100,000 \times 100,000$. Such matrix size is too large for a standard serial numerical routines like LAPACK to handle. On the other hand there is no parallel version of the LAPACK routines which can be used to solve the general eigenvalue problem. Furthermore, in our case, we are only interested in a very small portion of the eigenstates. Thus it might not be most efficient to use the direct methods. We have thus decided to use the PARPACK library ${ }^{7}$ which is a parallel library based on the iterative Arnoldi/Lanzos method. The detail of this method will be explained in Sec. III. One additional advantage of this iterative method is that the large Hamiltonian matrix $H_{\mathbf{G}, \mathbf{G}^{\prime}}^{\mathbf{k}}$ does not need to be written down explicitly, instead we only need to apply the Hamiltonian $H$ to a wave function, as a result the conventional methods such as the fast Fourier transform can be used to speed up this calculation.

According to previous studies on complex band structure, we know that if one draws a horizontal line for a given real energy $E$ in the graph of a band structure, the number of band structure lines (both real and complex) crossing this horizontal line will not change as the energy $E$ raises from the bottom. As a result, the complex branches of the band structure lines start from the valley points of the real band structure $E_{n}(\mathbf{k})$, where $d E_{n}(\mathbf{k}) / d \mathbf{k}=\left.0\right|_{k=k_{e}}$. Here the subscript $e$ stands for evanescent states. For the systems we are studying ( $\mathrm{Au}$ and $\mathrm{Cu}$ ) this occurs at the $\mathbf{k}=0$ ( $\Gamma$ point) and at $\mathbf{k}=\pi / a$ ( $X^{\prime}$ point), where $a$ is the unit-cell length in the $z$ direction (6.822 a.u. and 7.790 a.u. for $\mathrm{Cu}$ and $\mathrm{Au}$ respectively). This also happens when two real bands anticross each other and form a gap in the middle of the band structure zone. The complex band originated at $\Gamma$ and $X^{\prime}$ points will be called first and second imaginary bands respectively. The complex bands lying in the anticrossing gap will be called "generalized complex bands". The first imaginary band runs downward starting from a $\Gamma$ point on the real band structure. The second imaginary band and the generalized complex bands form close loops connecting two real $\mathbf{k}$ states at the opposite edges of an energy gap. A detail description of the complex band structure can be found in Refs. 1 and 9 .

Within the calculation scheme described above, the first and second imaginary bands can be obtained by first fixing $\mathbf{k}$ at 0 or $\pi / a$, respectively. Once $\mathbf{k}$ is fixed the imaginary $\kappa$ axis can be scanned using the Newton's method for zero $\operatorname{Im}(E)$ and the real energy eigenvalue is used as the complex band states.

The situation becomes more complicated for generalized complex bands. These bands lie between two anticrossing states. For this case we first find the maxima and minima of the real band structure. The minimum points are matched with the maximum points one by one by calculating the overlaps between their Bloch wave functions. We dont want to scan the whole $\{\mathbf{k}, \kappa\}$ space, that would be very expensive. We need to have a reference to know where the $\{\mathbf{k}, \kappa\}$ should be. The center of $k$ for the complex band structure branch is at $k_{c}=\left(k_{\max }+k_{\min }\right) / 2$. But we do not know what is the value of $\kappa$. To help us to find $\{\mathbf{k}, \kappa\}$, we have developed the following model to describe the complex band structure for the anticrossing 
case. The two bands involved in an anticrossing can be described by a $2 \times 2$ model Hamiltonian:

$$
H=\left(\begin{array}{cc}
C_{1} k & a \\
a & -C_{2} k
\end{array}\right)
$$

This Hamiltonian describes the bands for both the real band structure (real $k$ ) and complex band structure (complex $k$ ). The $C_{1}$ and $C_{2}$ are the slopes of the asymptotes of the parabolas lines passing through the center point, (see Fig. (1) and $a$ is half of the size of the gap.

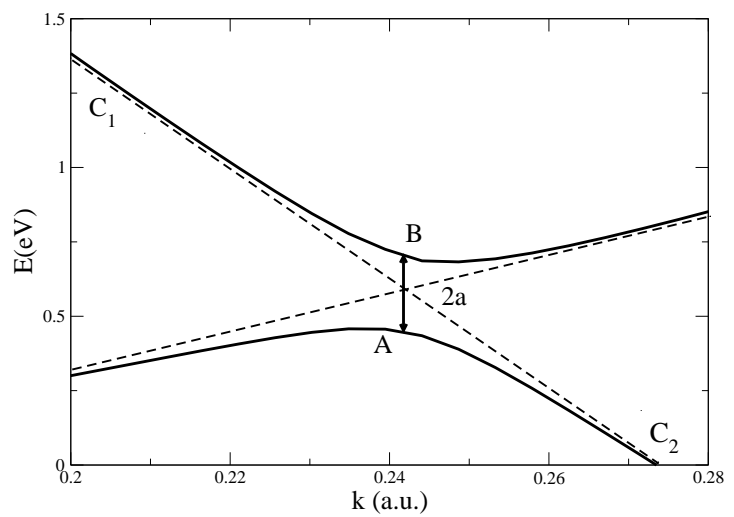

Figure 1: Plot of the energy states of the real band structure of a $\mathrm{Cu}(001)$ electrode, these states are represented by thick solid lines. The dashed line are the asymptotes of the states in the anticrossing gap where complex bands exist, $2 a$ is the gap between the lower and upper band lines.

Diagonalizing this matrix by setting $\operatorname{det}(H-E)=0$ yields the following equation

$$
\begin{aligned}
E=-\frac{1}{2}\left(C_{2}-\right. & \left.C_{1}\right) k \pm \\
& \sqrt{\frac{1}{4}\left(C_{2}-C_{1}\right)^{2} k^{2}+\left(C_{1} C_{2} k^{2}+a\right)} .
\end{aligned}
$$

Here the $k$ is complex. Thus we have: $k=k_{R}+i \kappa$. In general, we also have complex energy: $E=E_{R}+i E_{i}$. We are looking for solutions where $E_{i}=0$. If we plot $E_{R}$ and $E_{i}$ as functions of $k_{R}$ and $\kappa$, we get Fig. (2) and Fig. (3) repectively. We can identify the maxima (point A) and minima (point B). From Fig. (3), we first see a straight (dashed) line at $\kappa=0$ which gives us $E_{i}=0$. This corresponds to the real band structure (the solid line in Fig. (1) and the the thin black lines in Fig. (2)). Now we also see a loop which gives $E_{i}=0$ in Fig. (3). This loop (thick black lines in Fig. (2) and Fig. (3)) corresponds to the complex band structure. Guided by theses pictures, it is easy to search for the values of $\kappa$ of associated with a given $k$ that gives the real $E$ from Eq. (14).

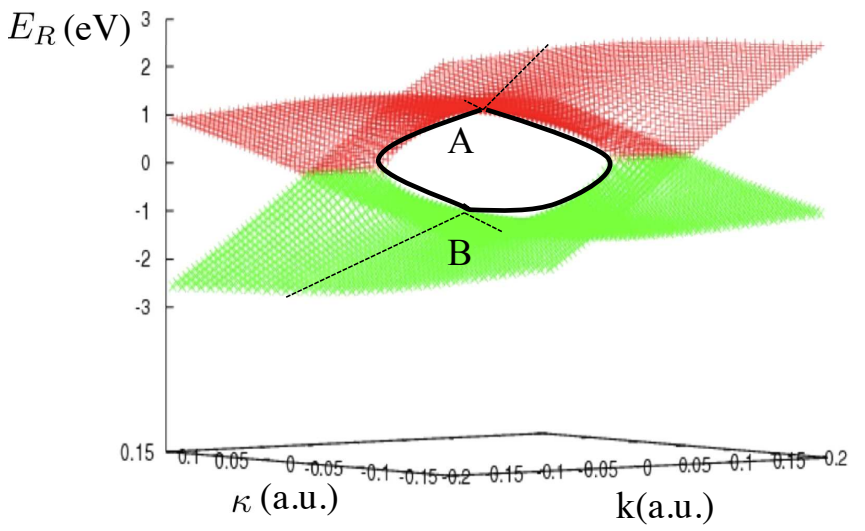

Figure 2: Plot of the real part of the Energy $E_{R}$, in function of $k_{R}$ and $\kappa$. The dashed line in the surface above the point $\mathrm{A}$ represents the $n=11$ energy state and the one in the surface behind the point $\mathrm{B}$ represents the $n=5$ energy state, both of the real band structure of $\mathrm{Cu}(001)$. The thick solid line represents the first few points with a $\kappa \neq 0$ but $E_{i}=0$.

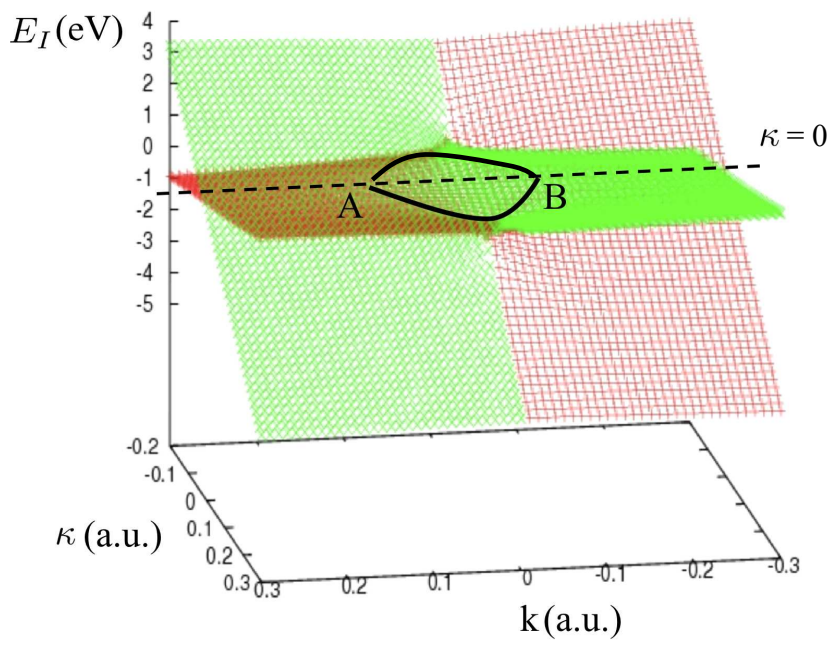

Figure 3: Plot of the imaginary part of the Energy $E_{i}$,in function of $k_{R}$ and $\kappa$. The point A represents the minima of the $n=11$ energy state and the point $\mathrm{B}$ the maxima of the $n=5$ energy state, both of the real band structure of $\mathrm{Cu}(001)$. The thick black line represents the solution of the Hamiltonian for a $\kappa \neq 0$ but $E_{i}=0$. And the dashed line the $\kappa=0$ axis.

Once we have the pairs $\left\{k_{R}, \kappa\right\}$ we diagonalize the Hamiltonian of Eq. (7) and select the eigenenergy with small $E_{i}$ within a numerical cut off, and with $E_{R}$ within the antocrossing band gap. This procedure is very robust and general so that it can be applied to other Hamiltonian (e.g., tight-binding). For our plane wave calculations we can solve for any metallic system and we can afford large energy cutoffs. 


\section{IMPLICIT RESTARTED ARNOLDI METHOD (IRAM)}

PARPACK is used to compute selected eigenvalues and the corresponding eigenvectors of the general Hamiltonian $H$. PARPACK is a parallel implementation of the implicitly restarted Arnoldi (IRA) algorithm. ${ }^{10}$ In IRA, the standard Arnoldi procedure ${ }^{11}$ is used to project an $n \times n$ Hamiltonian matrix $H$ into a low-dimensional Krylov subspace of the form $\mathcal{S}=\left\{v_{0}, H v_{0}, \ldots, H^{\ell-1} v_{0}\right\}$, where $\ell \ll n$. This procedure produces what is called an Arnoldi factorization of $H$, which can be described by

$$
H V=V T+\varepsilon
$$

where $V \in \mathbb{C}^{n \times \ell}$ contains an orthonormal basis of $\mathcal{S}$, $T \in \mathbb{C}^{\ell \times \ell}$ is a upper Hessenberg matrix, and $\varepsilon$ is the residual.

If $\mathcal{S}$, or equivalently $v_{0}$, is chosen appropriately, desired eigenvalues of $H$ can be obtained by computing the eigenvalues of $T$. That is, if $T s=\theta s$, then $z=V s$ is an approximate eigenvector of $H$ with the same eigenvalue $\theta$.

The IRA speeds up the convergence of Eq. (15) by applying the implicitly shifted $\mathrm{QR}$ algorithm ${ }^{12,13}$ to $T$ to yield

$$
H(V Q)=(V Q)\left(Q^{*} T Q\right)+\varepsilon Q
$$

where $Q=Q_{1} Q_{2} \cdots Q_{p}$ and $T-\mu_{i} I=Q_{i} R_{i} \quad(i=$ $1,2, \ldots, p, p<\ell-1)$ are QR factorizations ${ }^{14}$ of the shifted matrices $T-\mu_{i} I(i=1,2, \ldots, p)$ for some appropriately chosen shifts $\mu_{i}$ 's.

It can be shown that (16) leads to a new Arnoldi factorization (update of Eq. (15)):

$$
H \hat{V}=\hat{V} \hat{T}+\varepsilon^{\prime},,
$$

where

$$
\left(H-\mu_{1} I\right)\left(H-\mu_{2} I\right) \cdots\left(H-\mu_{p} I\right) V e_{1}=\hat{V} e_{1} \gamma,
$$

for some scalar $\gamma$. Equation (18) suggests that the updated Arnoldi factorization (17) is equivalent to a factorization produced from a new starting vector

$$
\hat{v}_{0}=\left(H-\mu_{1} I\right)\left(H-\mu_{2} I\right) \cdots\left(H-\mu_{p} I\right) v_{0} .
$$

This can be very advantageous. If the shifts $\mu_{1}, \mu_{2}$, $\ldots, \mu_{p}$ are chosen to be close to the eigenenergies of the unwanted eigenstates, we can filter out these undesired spectral components from $v_{0}$, thereby making $\theta$ converges quickly to the eigenenergies we want to obtain. During the iterations, these undesired $\mu_{p}$ can be obtained via the eigenvalues of $T$ outside a pre-selected desired eigenvalue window.

The update described by Eq. (16) can be performed repeatedly until the Krylov subspace associated with Eq. (17) contains an invariant subspace associated with the desired eigenvectors of $H$.
In our calculation, we chose $\ell=100$ and $p=50$. The shifts $\mu_{i}$ are chosen to be eigenvalues of $T$ that have large real or imaginary part. We need 57 updates in the case of $\mathrm{Cu}$ and 84 updates in the case of Au. Because $\ell$ and $p$ are small compared to the dimension of $H$, the computational cost of IRA is dominated by the matrix vector multiplications of the form $y \leftarrow H x$, which is parallelized in out computation. The computational time is around 3 min per $k$ point in the case of $\mathrm{Cu}$ and $6 \mathrm{~min}$ per $k$ point in the case of Au. In Fig. 4 we can find a progression of inverse of the requested time as a function of the number of nodes.

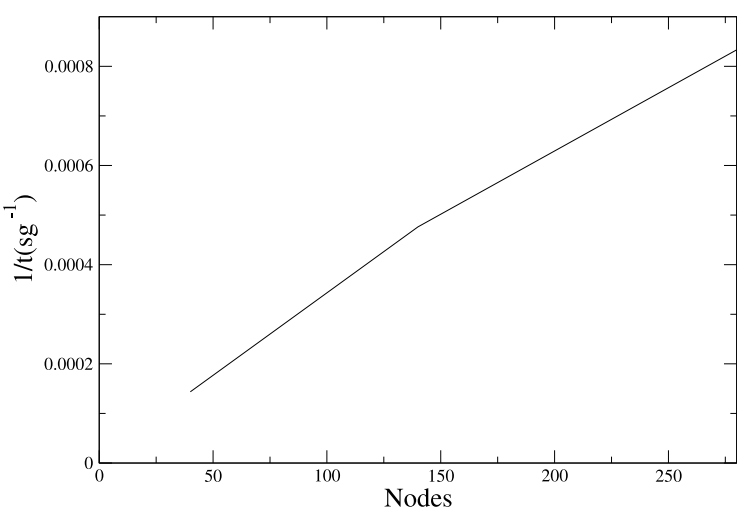

Figure 4: Plot of the inverse of the time requested to diagonalize the Hamiltonian matrix of the $\mathrm{Au}(001)$ at $\mathbf{k}=(0.5,0.02)$ a.u.

\section{RESULTS AND DISCUSSION}

We have tested three electrodes, all have been used in transport calculations. The first system is a $\mathrm{Cu}$ nanowire, as shown in Fig. (5) (in the lower right corner). The unit cell of the electrode has $9 \mathrm{Cu}$ atoms, but it is surrounded by a large volume of vacuum. The second electrode is a $\mathrm{Au}$ nanowire, with the same geometry of the $\mathrm{Cu}$ nanowire shown in Fig. (6). The third electrode is a bulk $\mathrm{Au}$ in (001) directions, with a cross section of $3 \times 3$ of the unit cells. It is shown in Fig. (7). For both $\mathrm{Cu}$ and $\mathrm{Au}$ atoms, we have treated the d electrons in the core. This is reasonable as the d electrons are completely occupied, and they are $2 \mathrm{eV}$ below the Fermi level. Thus they will not affect the transport in most experiments.

An energy cutoff $E_{\text {cut }}$ is used to select the plane-wave vector $\mathbf{G}$ entering Eqs. (4), (5), (6) and (12), where $\mathbf{G}=\left(\mathbf{G}_{\perp}, G_{z}\right)$ contains the components of the reciprocal vectors perpendicular and parallel to the direction of propagation $z$, respectively. If conventional spherical plane wave cut off method : $\frac{1}{2}|\mathbf{G}|^{2}<E_{c}$ is used, we will have a problem of describing the decay of evanescent states in $z$ direction. The exponential decay of the evanescent states in the $z$ direction cannot be described 
by a truncated plane wave basis set in that direction. As a result, we have used cylindrical plane-wave selection rule: the plane wave basis vectors will lie inside the cylinder $\frac{1}{2}\left|\mathbf{G}_{\perp}\right|^{2}<E_{c}$, and no cutoff is associated with $G_{z}$.

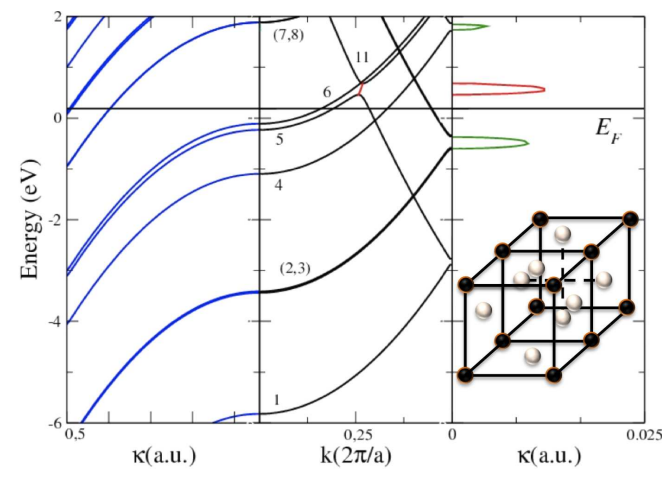

Figure 5: (Color on line) Complex band structure of $\mathrm{Cu}$ along (001). The Fermi energy of the electrode is at $0.189 \mathrm{eV}$. The nanowire is sourrounded with vacuum in the calculation.

Calculations are performed using norm-conserving pseudopotentials and a 30-Ry and 60-Ry cutoff for $\mathrm{Cu}$ and Au respectively.

The calculated complex band structures of $\mathrm{Cu}$ and $\mathrm{Au}$ wires are shown in Fig. (5) and (6) as well as the $3 \times 3$ electrode of $\mathrm{Au}$ in Fig. (7). The real band structure is represented in the middle panel. The lines in the left and right panels are the first and second imaginary bands, respectively. In the case of $\mathrm{Cu}(001)$ (Fig. (5)) the curve connecting bands 5 and 11 in the middle panel represents the generalized complex band lying in the $0.5 \mathrm{eV}$ gap of the real band structure. The imaginary part of this curve is shown as the red loop near the $X^{\prime}$-point vertical line. In the case of $\mathrm{Au}(001)$ (Fig. (6)) the generalized complex band lies between the 7,8 and 18,20 bands of

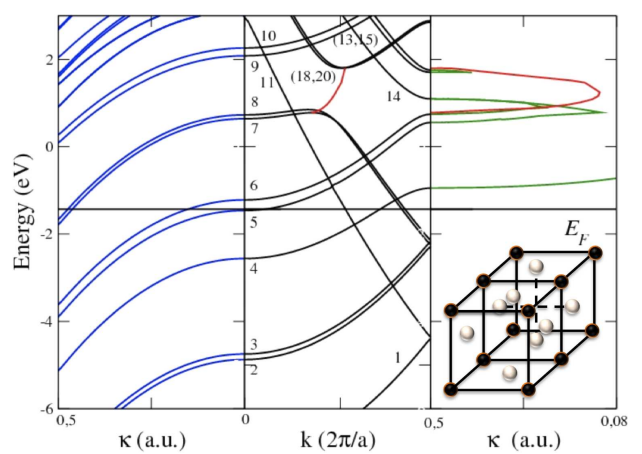

Figure 6: (Color on line) Complex band structure of Au along (001). The Fermi energy of the electrode is at $-1.431 \mathrm{eV}$. The nanowire is sourrounded with vacuum in the calculation.

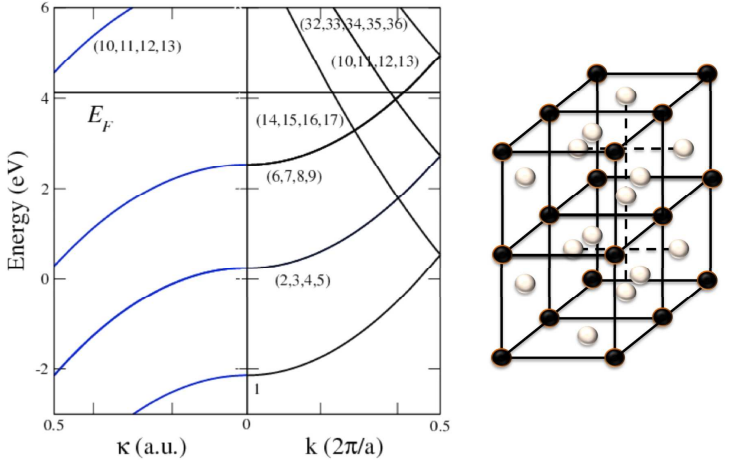

Figure 7: (Color on line) Complex band structure of $3 \times 3$ electrode of Au. The Fermi energy of the electrode is at 4.121 $\mathrm{eV}$.

the real band structure. In the case of $3 \times 3$ electrode of $\mathrm{Au}$ (Fig. (7)) we do not find any generalizing complex bands. There is no second imaginary bands at the $X$ ' point, either.

\section{SUMMARY AND CONCLUSIONS}

We have present a practical method to calculate the complex band structure of an electrode for quantum transport calculations. In this method, a generalized Hamiltonian is constructed for a given complex vector $\mathrm{k}$. The Hamiltonian is then diagonalized using implicit restarted arnoldi method as implemented in the PARPACK code. For typical electrodes used in quantum transport calculations, practical procedures are designed to search for the complex $\kappa$ which yield the zero imaginary eigenenergy $\operatorname{Im}(E)$. Especially for the generalized complex bands in the middle of the Brillouine zone, a two band anticrossing model is used to aid the search of the $\left\{k_{R}, \kappa\right\}$ point which yields real energy E. The use of this model can significantly improve the efficiency of this approach.

As there is no direct methods which yields the corresponding $\left\{k_{R}, \kappa\right\}$ for a given real energy $\mathrm{E}$ for plane wave Hamitlonian with nonlocal pseudopotentials, the approach presented in the current paper might be the only option. Our work indicates that it is quite practical to use this straight forward approach for most electrodes used in quantum transport calculations, provided that the iterative generalized eigensolver is used and the model is used to help the search of $\left\{k_{R}, \kappa\right\}$. This is particularly suitable for our auxiliary periodic boundary condition method to calculate the scattering states. In this method, only a few evanescent states with relatively 
small $\kappa$ are needed in the wave function decomposition analysis. Thus, unlike many other methods, there is no need to get all the evanescent states in the Hamiltonian for a given energy $E$. Thus, in the actual calculations, the band structures (including the real band structures and complex band structures) of an electrode is precalculated with a finite number of $k_{R}$ and $\kappa$ points (e.g., 100 points), and their energies and wave functions are stored. In the scattering state calculation for a given energy $E$, the relevant running wave and evanescent Bloch states (the states crossing a horizontal line of $\mathrm{E}$ in the band structure figures $5,6,7)$ are obtained via interpolation between the neighoring $k_{R}$ and $\kappa$ grid points which have already been calculated (and stored).

\section{ACKNOWLEDGMENTS}

This work has been supported partially by the U.S. Department Of Energy BES/SC under contract No. DEAC02-05CH11231 and the Gobierno Vasco Postdoctoral Fellowship DK program. This research used the resource of the National Energy Research Scientific Computing Center (NERSC).
1 Y.C. Chang, Phys. Rev. B 25, 605 (1982).

2 Y.C. Chang, J.N. Schulman, Phys. Rev. B 25, 3975 (1982).

${ }^{3}$ H.J. Choi, J. Ihm, Phys. Rev. B 59, 2267 (1999).

${ }^{4}$ L.W. Wang, Phys. Rev. B 72, 045417(2005).

5 A. Garcia-Lekue and L.W. Wang, Phys. Rev. B 74, 245404 (2006).

6 A. Garcia-Lekue and L.W. Wang, J. Comp. Mat. Sci. 45, 1016 (2009).

7 R. B. Lehoucq, D.C. Sorensen, and C. Yang, ARPACK Users Guide: Solution of Large-Scale Eigenvalue Problems with Implicity Restarted Arnoldi Methods, SIAM, Philadelphia PA (1998). http://www.caam.rice.edu/software/ARPACK/.

${ }^{8}$ L. Kleinman and D. M. Bylander, Phys. Rev. Lett. 48,
1425 (1982).

9 D. L. Smith and C. Mailhiot, Rev. Mod. Phys. 62, 173 (1990).

10 D. C. Sorensen, SIAM Journal on Matrix Analysis and Applications 13-1, 357-385 (1992).

11 W. E. Arnoldi, Quarterly of Applied Mathematics 9, 17 (1951).

12 J. G. F. Francis, The Computer Journal 4, 265 (1961).

13 J. G. F. Francis, The Computer Journal 4, 332 (1961).

14 G. H. Golub and C. F. Van Loan, Matrix Computations, Johns Hopkins University Press, Baltimore MD (1996).

15 https://hpcrd.lbl.gov/ linwang/PEtot/PEtot.html 